\title{
ERYTHEMA EXUDATIVUM MULTIFORME IN CHILDHOOD
}

\author{
BY \\ A. V. NEALE, M.D., F.R.C.P. \\ (From the Department of Child Health, University of Bristol)
}

Hebra (1860) entered into a friendly rivalry with the English dermatologist Robert Willan in his classical dissertations on the "polymorphous erythemata.' Even in these early years dermatological nomenclature had reached a complex state. Hebra comments that 'these various names by no means answer to as many distinct diseases, and our object must be to determine which of them apply merely to appearances developed in succession during the course of one and the same disease, and, in reference to this, experience has taught me that E. papulatum, E. tuberculatum, E. annulare, E. iris, E. gyratum are merely forms of the same disease according to the period of its course; to this malady I shall apply the name E. multiforme.' The phases and transitions are clearly described by Hebra and some emphasis is placed upon the regional distribution of the rash, it appearing initially on the dorsum of the hands and feet, being in severe cases more generalized even to include, in exceptional cases, the trunk and face. In Hebra's description E. papulatum represents the lowest and E. gyratum the highest grade in the eruption, and he deprecates the notion that these are different species. Also, E. exudativum multiforme may give rise to trifling subjective disorder, fever being observed only in exceptional cases and in those in which the affection spreads over large tracts of the surface and having a duration of up to four weeks. Hebra agrees with Willan that vesicular forms of the eruption may appear, but he refuses to agree that there is any relation with Herpes; and he concludes his masterly clinical description in confessing his complete ignorance of the cause of these erythemata, although his experience indicated a preponderance of these affections in young persons.

It is of some historical interest in this connexion that Hebra (1860) discusses E. epidemicum, aerodynia (which Alibert says prevailed in Paris in 1828), and classifies it as an exudative erythema. Two years later, Bazin (1862) described vesiculo-bullous stomatitis in association with multiform cutaneous lesions.
It is evident that in the following years there was much dispute in regard to the classification and etiology of the erythematous dermatoses. We now understand that individual susceptibility and reaction is indicated by a wide variation in clinical response, and this is specially so in the cutaneous and mucosal surfaces, which in their display of colour, form, shape, and distribution rarely fail to demand careful clinical scrutiny. Whilst, therefore, for certain practical reasons, many of the skin reactions arising out of general disease retain their original and historical descriptive names (which may or may not bear any clear relation to the known etiological factors in the particular case), it has not been entirely favourable in general observation in disease. Dermatological aspects have inclined to dominate description or to cause publication of cases of more general interest in relatively isolated and specialized literature. This trend in using terms according to local signs has undoubtedly led to considerable confusion, and this is specially pointed out by Levy (1947) in his observations on the 'Stevens-Johnson' syndrome. In this, a group of signs and symptoms have received widely divergent names according to the viewpoint of the observer, or to certain variations in clinical character in individual cases. There is undoubtedly reasonable cause to gather together a group of disorders wherein particular cutaneous and mucosal responses are variably associated, and whilst the ingenuity of some of the names employed, for example dermatostomatitis, mucosal respiratory syndrome, mucocutaneous fever, etc., is recognized, none appears to be sufficiently definitive for continued use. It seems advisable to retain the name $E$. multiforme and to qualify the term according to additive phenomena. As Stannus (1947) points out, there are wide clinical variations in the disease, and the Stevens-Johnson syndrome belongs to or is part of the condition usually regarded as $\mathrm{E}$. multiforme. Whilst Hebra in 1860 regarded E. multiforme as a primary cutaneous disease with relatively benign effects, it is clear from the literature since his day 
that associated visceral pathology is not unusual and that severe illness is by no means rare. Fiessinger observed the syndrome characterized by a vesiculo-bullous eruption involving the mucous membranes, including the anal and genital regions and associated with fever and leucopenia.

Stevens and Johnson (1922) claim description of a - new eruptive fever associated with stomatitis and ophthalmia ': the notion that it is new is probably unreal in the light of the historical facts outlined by Keil (1940) and mentioned by Levy (1947). However, no doubt Stevens and Johnson were so very impressed by the startling clinical appearances in their two cases in children that they were convinced of their unique character. In their clinical summary they note the generalized cutaneous eruption ' not conforming to any recognized dermatologic condition,' and in both cases there was a purulent conjunctivitis. In one of the boys this proceeded to panophthalmitis and total loss of vision. In the other boy severe corneal scarring resulted. A high and continuous fever was present in each case, with evidence of pulmonary involvement in one child but not so in the other. The onset was with fever, and the eruption showed identical characteristics in each, the rash appearing on the back of the neck and the chest, spreading to the face, arms, and legs during a period of about eighteen days, the last lesions to appear being on the soles and the palms at about the time when the first eruptive zones were showing resolution. The eruption showed oval dark red to purple macules which in a few days were raised firm brownish purple papules 0.5 to $2 \mathrm{~cm}$. in the longest diameter, without pain or pruritus. Some foci may reveal a yellow necrotic centre. On the forearms and shins the lesions were smaller but more thickly disposed. There were no pustules. The scalp was uninvolved, but the mouth and lips were intensely sore and inflamed, and bullae appeared early on the oral mucosa. Resolution of the skin lesions was evident after three weeks, and residual faint pigmented areas, without pitting or scarring, were seen. Fall of temperature coincided with the period of resolution which finally involved the forearms and legs six weeks from the onset.

Baader (1925) observed dermato-stomatitis with pseudodiphtheritic ulceration of the lips and sublingual surface and similar scrotal erosion. Forman and Whitwell (1934) noted that herpes simplex often precedes an attack of $\mathrm{E}$. multiforme. They suggest that a virus infection may be the cause of E. multiforme of the Hebra type.

Ageloff (1940) stresses the possible ocular manifestations. In his cases of E. multiforme bullosum, severe involvement of the eyes called for urgent selective treatment. In girls, vaginitis may be present. Schlesinger (1904) considered that the disease is relatively more severe in children.

We are indebted to Keil (1940) for a comprehensive historical and clinical survey of E. multiforme, wherein he notes that the variable clinical course and the complicated nomenclature has tended to create an artificial hiatus between internal medicine and dermatology. Keil regards Hebra's E. multiforme exudativum as a specific entity. The term E. multiforme is used to designate erythema-papular rashes of acute onset and brief course and having special favour for the extensor aspect of the limb, to which may be added vesicular or pustular components. The vesiculo-bullous type is more often seen by paediatricians and ophthalmologists, whilst the ordinary erythematous case runs its course with minimal subjective complaint. Involvement of mucous membranes indicates a more severe type of the disease, with correspondingly increased general effects and eruptive fever. Gross haemorrhagic bullae with widespread lesions may render it difficult to recognize the ordinary features of E. multiforme exudativum. The dangers of the disease are in proportion to the mucous membrane involvement. Superficial erosion in the mouth may be the only feature. In the eyes, secondary infection may, similarly, change the appearances. In the mouth the initial erythematous phase is quickly followed by vesicles and bullae which may extend to the palate, pharynx, larynx, tracheobronchial, and oesophageal areas, thus creating a dangerous condition. Involvement of the conjunctivae and possibly the cornea appears to follow the onset of the exanthema; pain and photophobia are succeeded by considerable swelling of the eyelids and a muco-purulent discharge. Ocular vulnerability is particularly prominent in children, the lesions varying in depth from a superficial catarrhal change to purulent reaction in which the bulbar structures may be affected. A danger exists of panophthalmitis. In the severe ocular cases there is invariably involvement of the oral and sometimes other mucosal surfaces and the cutaneous tissues. In discussing the Stevens-Johnson syndrome Keil regards it as a variant of $E$. multiforme exudativum with severe ocular lesions and that children are predisposed to the graver forms. Also exudative reaction may occur on the glans penis, prepuce, urinary meatus, urethra, labia, vaginal tract, cervix, anal canal, and the lower rectum; and ulcero-necrotic areas may quickly spread on the scrotum. Urethral reaction may be severe, and it is possible that some cases of non-specific urethritis are in this group. The literary term ' ectodermosis erosiva pluri-orificialis' (Fiessinger and Rendu, 1917) represents variants of the $E$. multiforme exudativum syndrome in regard to the distribution of the mucous membrane lesions.

Dowling (1940) observed during 1939 six cases of a 'pemphigus-like eruption.' His description of one of the cases is specially interesting and typical of the group. 'A child of five years developed conjunctivitis followed in a few days by severe stomatitis and almost immediately afterwards small bullae appeared all over the skin. The child appeared severely though not dangerously ill, with 
pyrexia. There was considerable conjunctivitis and the mouth and tongue were covered with superficial ulcers and sloughs, and the lips swollen. Blisters appeared on the penis and scrotum and similar lesions all over the surface, in largest numbers on the face and extremities. The temperature declined steadily and after eleven days' illness the child recovered.' Dowling regards E. multiforme as not a rare type of cutaneous reaction in the epidemics of winter sore throats but that the clinical severity in this particular group was unusual and the appearance of large numbers of bullae suggested pemphigus; even variola was suspected.

Raffetto and Nichols (1942) suspected that sulphadiazine was an aggravating factor in a girl aged ten years who developed, eight days after acute tonsillitis, generalized vesiculo-bullous eruptions with oral and conjunctival lesions. In this case there was 20 per cent. eosinophilia.

A similar but fatal case with oral necrosis is recorded by Dardinski (1945). Soll (1947) concludes from observations on twenty cases that sulphonamides should be avoided owing to the eruptive manifestations. Phenobarbitone is regarded by Moss and Long (1942) as a possible cause of severe toxic eruptions affecting the skin and the mucosal surfaces and closely resembling E. multiforme exudativum.

Of nearly 72,000 patients in a large infectious diseases hospital in Manhattan, twenty-eight cases of $\mathrm{E}$. multiforme bullosum were recognized, all showing multiform erythematous cutaneous lesions, bullous formations in the skin and mucous mombranes, and constitutional symptoms compatible with acute infectious disease. Most cases were children under ten years of age. Fever, sore throat, headache, vomiting, and anorexia, together with ocular, gastro-intestinal, respiratory, and genitourinary symptoms were present. The principal cutaneous lesions were dark red macules and papules proceeding to vesicles and large bullae. To a less extent generalized erythema, pustules, purpura, crusted lesions, desquamation, and iritis occurred, and conjunctivitis appeared in moderate degree. There was some evidence of concurrent respiratory infection, and three cases were fatal. In this series Fletcher and Harris (1945) give special consideration to differential diagnosis. Considerable confusion was evident in the pre-admission diagnosis, haemorrhagic varicella or variola being suspected, and only one case in the whole series was correctly diagnosed before admission to hospital.

The bizarre nature of the disease is further illustrated by the studies of Stanyon and Warner (1945), who under the term 'mucosal respiratory syndrome' describe prostrating febrile illness with mucosal involvement as conjunctivitis, membranous stomatitis, dermal eruptions, urethral lesions, and pneumonia. The initial symptoms were like influenza, with upper respiratory infection followed by lung lesions resembling the common atypical pneumonia. Bacteriological studies were essentially negative, although a virus was isolated in two cases. Of great interest is the fact that cutaneous eruption was present in only about half the cases, although there was no reason to doubt the common etiology in the series. When present, the skin lesions were very distinctive, being confined to the forearms, dorsum of hands and feet, legs, and scrotum. The stomatitis was usually an early and dominant symptom. In their fatal cases these authors found a certain distinctive pathology in the viscera and buccal mucosa but no specific infective agent could be isolated. The evidence was suggestive of a virus disease; attempts to transfer the disease to animals were unsuccessful. The Commission on Acute Respiratory Disease note also the prevalence of non-bacterial pneumonia, with essentially mononuclear reactions, in either the erythemato-papular or the vesiculo-bullous forms of $E$. multiforme exudativum.

In New York in 1947 (Williams) a considerable increase in the incidence of $E$. multiforme was seen after vaccination. In these cases there was inconspicuous oral and no conjunctival lesion. The usual interval was ten days. This phenomenon was also observed by Schwartz and Brainerd (1946) to follow five cases of smallpox vaccination, one case of tetanus toxoid injection, and in one case after injecting influenza virus vaccine. In such patients the $E$. multiforme response occurred at an interval of ten days. These observations lend some support to a suspected virus-allergic relationship in $E$. multiforme.

\section{Diagnosis}

From the descriptive literature as first outlined by Hebra (who apparently did not record the presence of mucosal lesions) and later more elaborately discussed by a long series of observers, a picture of the bizarre manifestation of this disease may be obtained, but direct inspection of the patient is the only means of acquiring a clinical memory of the remarkable appearances usually presented. As Keil relates, the clinical pictures may be dominated by systemic disorder, varying as a rule with the intensity of the muco-cutaneous effects. Initially an upper respiratory coryzal infection or a sore throat, mild tonsillitis, pharyngitis and laryngitis, and a local lymphadenopathy may precede the exanthema by a period up to two weeks. Painful throat, dysphagia, and foetor oris may supervene. Severe coughing and possibly blood-tinged sputum may follow. The external areas of the nasal passages may be intensively irritated, and accompanied by discharge. Pyrexia may range to $105^{\circ} \mathrm{F}$., and even the papular forms of the disease may be associated with considerable pyrexia. No cardiac changes have been detected. Gastrointestinal effects are minimal, and abdominal examination is usually negative. Eosinophilia has 
been recorded in a few cases, but there are usually no significant haematological findings. Haematuria may be due to erosive effects of the urethral and preputial vesicular ukeration.

Respiratory infection may arise as an extended disorder from the oral and nasal ulcero-necrotic changes or, as specially noted in the Canadian cases, primary consolidating disease in lungs may be present of a type resembling atypical pneumonia with mononuclear cell reaction.

Soll (1947) considers that cases may be divided into three groups based upon the severity and distribution of the lesions (a) as originally described by Hebra and including mild forms primarily involving the skin and showing minimal systemic disturbance or mucosal effects, (b) an extremely severe form with extensive lesions on the mucous membranes, cornea, conjunctiva, and ominous possibilities of panophthalmitis, (c) an intermediate type with most effects visible upon the mouth and other mucous membranes and a special tendency to urethritis and balanitis. Nellen (1947) relates a very characteristic example of erythema exudativum multiforme bullosum with ecchymotic conjunctivitis, stomatitis, and urethritis, and in which, after one week's illness with high fever, vesicles on an erythematous base appeared over the extensor surfaces of the arms and legs and soon the patient presented a florid picture, pus pouring from the eyes, mouth, and urethra.

The ophthalmological manifestations in $E$. exudativum multiforme may vary from mild hyperaemic changes to a severe pseudomembraneous form in which disastrous results may arise out of corneal ulceration. There is usually considerable swelling of the eyelids with oedema and vesicle formation, and this is well illustrated in Nellen's paper. Duke-Elder (1938) notes that Fuchs (1876) first recorded the ocular involvement, or ' herpes iris conjunctivae,' in a case which showed typical skin and oral lesions.

In a recent paper, Sneddon (1947) agrees that the eruption at the maximal phase of the disease may be generalized, but usually in the early stages the extremities, including the palms and soles, are involved, macules and papules being followed in the more severe cases by bullae. He notes that the classical target and erythema iris lesions were uncommon in his cases and that, unlike the common erythema multiforme, relapse is unusual. Subsequent attacks, however, may appear with months' or years' interval and with any or all of the previous signs, but usually with a lessened intensity. Membraneous sloughing in the mouth may be particularly severe, as in the two boys reported by Murray (1947).

\section{Etiology}

The disease has many of the features of a primary infection. Acute upper respiratory inflammatory disorder may open up channels of entry and an interval of up to two weeks in such cases may represent an incubation period or a phase of latency in the development of cutaneous and mucosal susceptibility. The association of a virus type of pulmonary lesions in some of the cases is probably very significant and is supportive of the growing evidence in relation to virus infection in erythema exudativum multiforme. Attempts to transfer the disease to animals have so far been unsuccessful. It is significant that in their fatal cases Stanyon and Warner (1945) found in the visceral and buccal mucosa a distinct pathology suggestive of a virus disease, but no specific infective agent could be isolated. The occasional appearance of the syndrome after toxoid or vaccine injection suggests infection and allergy as operative agents. Biopsy of the skin by Nellen revealed non-specific inflammatory changes. Vesicle fluid gave negative reactions for variola and vaccinia antigens (Murray). Much of the primary pathology, especially in the mucous membranes, may be quickly changed by secondary infection, and more especially with staphylococci. Probably extended study with viruses will yield more positive information.

Ease 1. A boy aged four years had had no previous illness. An attack of mild respiratory disorder with sore throat had begun two weeks previous to the appearance of 'spots on the legs,' which were shortly followed by a similar eruption on the legs, face, chest, and back. The wrists became painful. In another twenty-four hours the rash became haemorrhagic. The mouth was sore, reddened, and very oedematous, and there was some blood-stained mucous in the throat. The clinical picture on admission to hospital was somewhat startling and clearly indicated an unusual disorder. The boy had a pallid, puffy face with considerable oedema; suffusion and haemorrhagic eruptions were present in and around the conjunctivae. The mouth and lips were very swollen, intensely sore, and discharging haemorrhagic secretions. The tonsils and pharynx were hyperaemic. On the face, arms, feet, buttocks, and thighs a haemorrhagic papulo-bullous eruption was present, the maximum distribution of the rash being rather peripheral and chiefly on the extensor aspects. There was pain and swelling of the joints, especially the knees and wrists, and moderate effusion appeared in the left knee. There were no abnormal cardiac or pulmonary signs, and the nervous system was normal. The spleen was not enlarged, and abdominal examination was negative. Blood examination showed no abnormality apart from a slight neutrophil leucocytosis. There was no eosinophilia. 


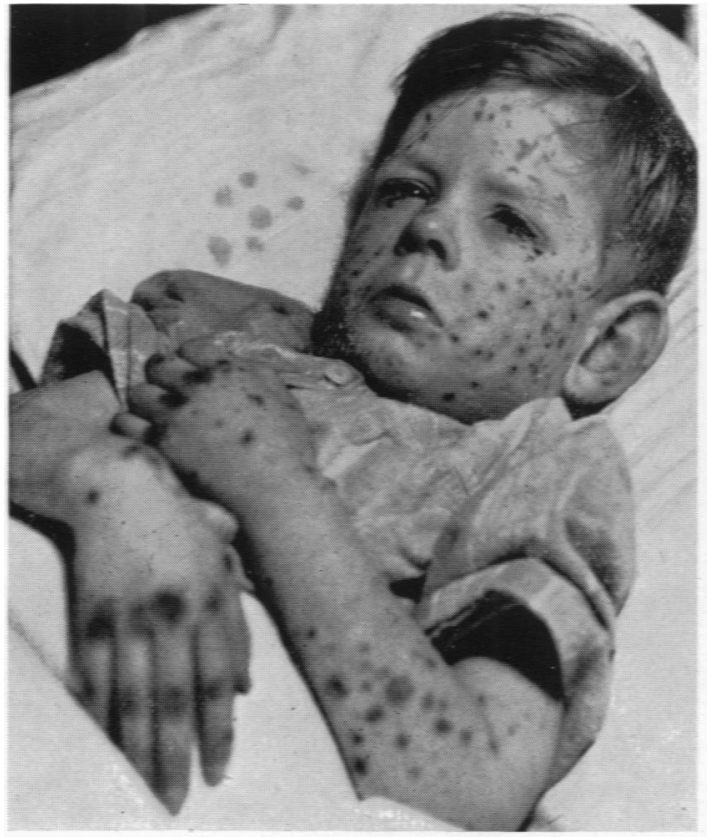

FIG. 1.

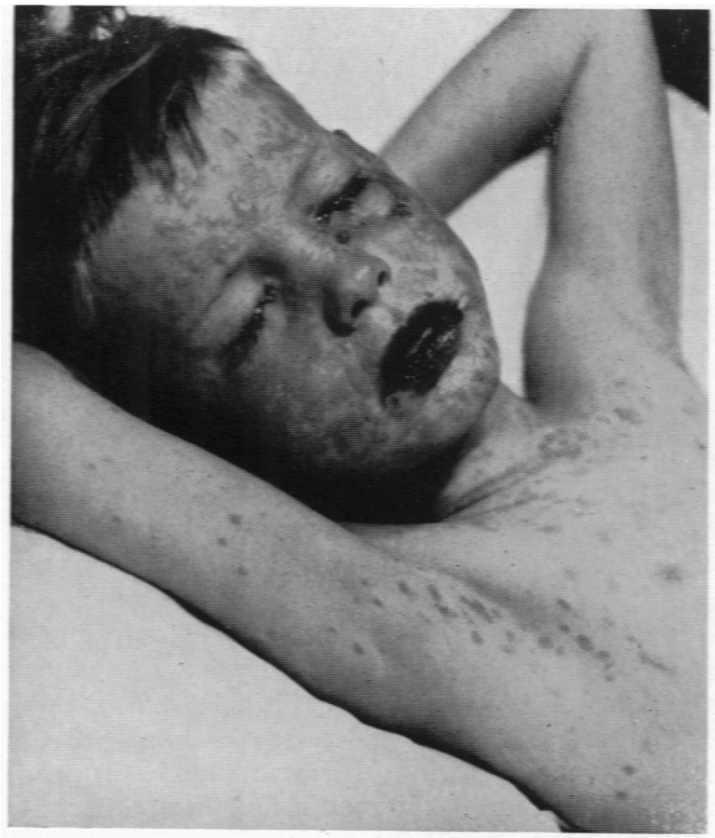

FIG. 3.

Figs. 3 and 4.-Maximum phase of the eruption, Case 2. Note confluence and vesicular formation on the face and forehead.

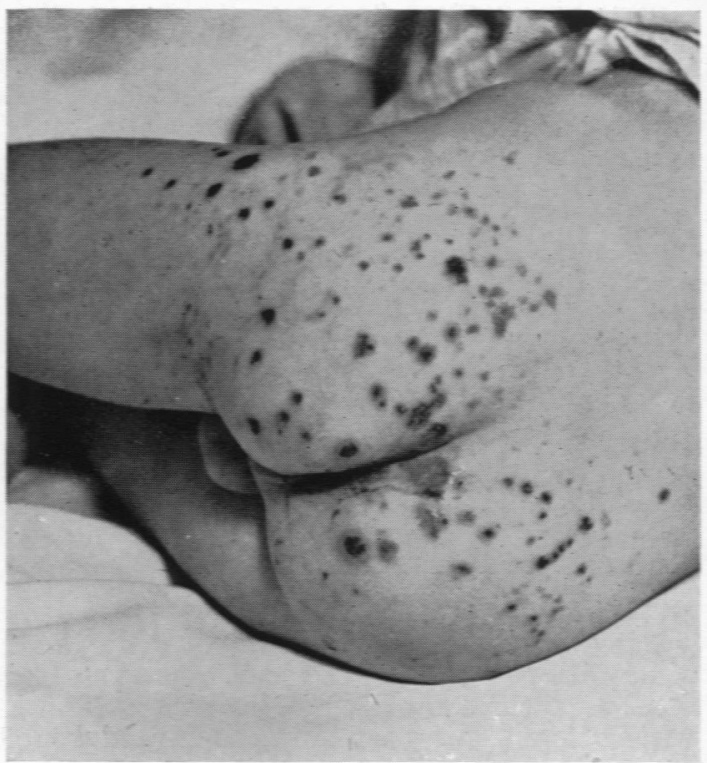

FIG. 2.

Figs. 1 and 2.-Maximum phase of the eruption, Case 1.

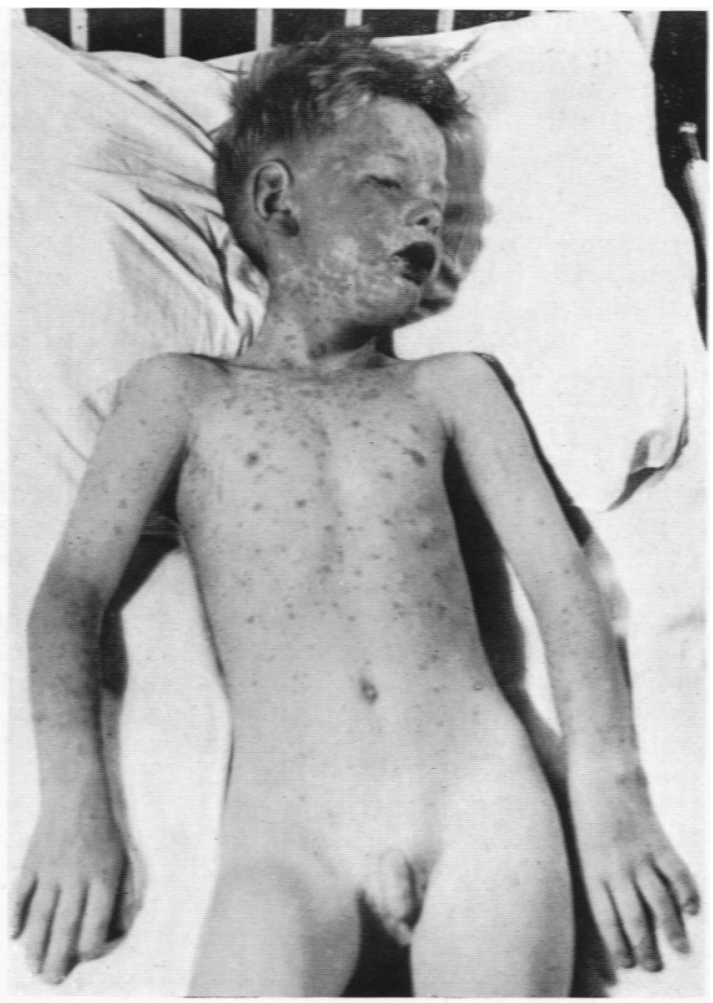

FIG. 4. 
During the following week the rash did not extend, but at points of decubitus pressure a deepening necrosis threatened in some of the haemorrhagic areas, especially on the olecranon and gluteal parts. Anything more than temporary pressure indicated a special susceptibility to subcutaneous tissue damage. The swollen joints gradually subsided in about ten days. No nodular swellings occurred. Fortunately there was no spread of the conjunctival eruption to the ocular structures, and although the facial swelling cleared slowly the clinical course of the illness was markedly abating after fourteen days. A mild, simple albuminuria quickly cleared. This boy had no signs of urethral or genital affection, although it is possible that a transient melaena occurred in the earliest phase. Penicillin, 15,000 units three-hourly, was given over a period of ten days, with the primary object of preventing or combating secondary coccal sepsis. (See figs. 1 and 2.)

Case 2. A boy aged seven years entered into an acute illness with high fever, restlessness, and moderate delirium. His eyes and mouth quickly became sore. Dysphagia and cough were troublesome. Difficulty in micturition was associated with acute bullous eruption in the visible urethra and prepuce, with considerable local swelling. Five days from the first symptoms the boy was intensely miserable, the eyes; mouth, and skin being distressful, and in fact all the visible mucous membranes were in acute exudative eruption. The clinical impression was, however, that the boy was not dangerously ill, as he appeared unexpectedly alert on occasions. The skin showed erythematous patches varying in size from $\frac{1}{2}$ in. to $\frac{1}{2}$ in. and of irregular shape with central vesicle, and being principally disposed on the limbs and the face, the scalp and back being significantly free. Both anterior and posterior surfaces of the limbs were affected and a few papules were present on the hands and feet. Most of the skin lesions blanched on pressure. There was bilateral purulent conjunctivitis with the lids encrusted with thick purulent exudate, but the cornea and other ocular structures did not appear to be involved. Some bleeding foci were seen in the nasal mucosa, and the mouth presented membraneous sloughs, especially on the inner cheeks and tongue, which bled easily on local swabbing. There was no visceral abnormality. Blood count was quite normal, and there was no eosinophilia. After six days the exudative reactions showed early resolution, but dysphagia proved troublesome owing to the pain and immobility of the oral structures. Penicillin, 20,000 units at three-hourly intervals, was given as a protective measure, although no pyogenic bacteria were found in the nasal or oral material. (See figs. 3 and 4.)

\section{Discussion}

The varied and somewhat bizarre descriptions surrounding the disease type initially recorded by
Hebra are an indication of the many ways in which clinical description may differ when dominant manifestations may be present in distinctive tissues. The 'scattering' in medical literature is no doubt due to the variety in leading signs, which is probably in reference to the factors of primary virus infection, special tissue reaction (especially ectodermal), anaphylactic-like phenomena, and secondary sepsis. In the form of erythema under review some importance is attached to clinical accuracy, especially in regard to possible ocular, oral, or cutaneous resemblance to other conditions. Special difficulty may arise when, as is sometimes the case, the skin effects are minimal and the clinical state is essentially that of acute involvement of the mucous membranes of the mouth and of the conjunctiva. Panophthalmitis with perforation of the eye must be regarded in nearly all cases as the worst possible danger, and especially so in young children. The absence of vesicular or bullous changes and the quick variations in distribution should distinguish E. marginatum. Polymorphous rashes, usually about joints, but sometimes with a more generalized tendency and a secondary haemorrhagic appearance with minute vesicles, are observed in the Henoch-Schönlein syndrome. It is of considerable interest that, in one case, joint swellings were recognized during the acute phase of the illness. There was, however, complete absence of any abdominal symptoms.

The condition of the membranes of the mouth may cast a possible suspicion of diphtheria, although the bullous changes in the mucous membranes and lips clearly dispose of the possibility.

Perhaps some resemblance may exist in regard to variola, and in fact confusion has occurred occasionally. Certain atypical forms of smallpox may have to be considered when papular lesions, however few, quickly acquire vesicular, pustular, or haemorrhagic changes, and especially when such lesions are disposed more particularly about the face, forehead, backs of the hands and feet, and the wrists. Increasing familiarity with the syndrome of E. multiforme of the type discussed in this paper should, however, soon dispose of essential difficulty. Although many of the patients pass through their illness without significant danger, careful watch must be kept on certain possibilities, and probably in the order of importance as (1) conjunctival disease spreading to the ocular structures, (2) progressive ulceration in the mouth favouring descending respiratory sepsis, (3) pressure effects on the skin leading to a deepening necrosis.

Treatment. It is in relation to these more severe possibilities that treatment is particularly directed to the affected mucous membranes and the eyes. 
Whilst penicillin probably has beneficial action against secondary staphylococcal infection, sulphonamides are better avoided in view of the aggravating effects which have been strongly suspected in some of the cases reported. Neller noted that sulphamezathine was without favourable effect in the severe phase, but general improvement followed penicillin intramuscularly. In a boy bearing the clinical features of $E$. multiforme bullosa, with marked oral and ocular signs, the disease was apparently aggravated by sulphathiazole, and, in fact, this was considered to be the determining factor in the fatal termination. Nicotinic acid therapy is considered favourably by Weisberg and Rosen (1946).

The photographs in this paper were taken by Mr. J. Gregory Williamson, to whom I am extremely grateful.

\section{REFERENCES}

Ageloff, H. (1940). New Engl. J. Med., 223, 217.

Baader, E. (1925). Arch. derm. syph., Wien., 149, 261.

Bazin, E. (1862). Leçons sur les affections de la peau. Paris.

Commission on acute respiratory disease (1946). Arch. intern. Med., 78, 687.

Dardinski, V. J. (1945). Amer. J. clin. Path., 15, 28.

Dowling, G.B. (1940). Lancet, 2, 759.

Downing. J. G. (1947). New Engl. J. Med., 237, 946.

Duke-Elder, W. S. (1938.) Textbook of Ophthalmology, Vol. 2.

Fiessinger, N., and Rendu, R. (1917). Paris Méd., 23, 54.

Fletcher, M. W., and Harris, R. C. (1945). J. Pediat., $27,465$.

Forman, L., and Whitwell, G. P. B. (1934). Brit. J. Derm. Syph., 46, 309.
Haddad, N. N. (1945). J. Canad. med. Serv., 2, 657.

Hebra, F. (1860). In Handbuch der Pathologie, 3, 198. Virchow. R. Erlanger.

- (1866). Vol. 1, Diseases of the Skin. New Sydenham Society, London.

Hotston, R. N., and Pemberton, H. S. (1947). Lancet, $1,499$.

Keil, H. (1940). Ann. intern. Med., 14, 449.

Laurence, D. R. (1947). Lancet, 1, 888.

Leading article (1947). Ibid., 1, 374.

Levy, H. (1947). Lancet, 1, 537.

Lever, W. F. (1944). Arch. Derm. Syph., Chicago, 49, 47.

Moss, R. E., and Long, W. E. (1942). Ibid., 46, 386.

Mowbray, R. (1947). Lancet, 1, 538.

Murray, J. O. (1947). Ibid., 1, 328.

Nellen, M. (1947). Ibid., 1, 326.

Osler, W. (1895). Amer. J. med. Sci., 110, 629.

Raffetto, J. F., and Nichols, S. (1942). J. Pediat., 20, 753.

Schlesinger, E. (1904). Arch. Kinderheilk., 40, 256.

Schwartz, M. H., and Brainerd. H. D. (1946). J. Pediat., 29, 512.

Sears, W. G. (1947). Lancet, 1, 427.

Sheldon, J. H. (1947). Arch. Dis. Childh., 22, 7.

Sneddon, I. B. (1947). Brit. med. J., 1, 925.

Soll, S. N. (1947). Arch. intern. Med., 79, 475.

Stannus, H. S. (1947). Lancet, 1, 538.

Stanyon, J. H., and Warner, W. P. (1945). Canad. med. Ass. J., 53, 427.

Stevens, A. M., and Johnson, F. C. (1922). Amer. J. Dis. Child., 24, 526.

Weisberg, A., and Rosen, E. (1946). Arch. Derm. Syph., Chicago, 53, 99.

White, G. E. (1943). Canad. med. Ass. J., 49, 317.

Willan, R. (1799). Cutaneous Diseases. Breslau.

Williams, W. (1947). Private communication.

Year Book of Dermatology and Syphylology (1946). pp. 252 and 256. Chicago. 